\title{
Chapter 9 \\ The Biology of Breast Cancer Disparities in Hispanics: Current Knowledge, Gaps, and Research Opportunities
}

\author{
Gerardo Colon-Otero
}

\section{Introduction}

The Hispanic population in the United States has been steadily increasing over the last decades, and it currently represents over 54 million people or approximately $19 \%$ of the US population (U.S. Census Bureau data, 2014). Breast cancer is the most common cancer in Hispanics and also has the highest mortality; therefore, addressing disparities in breast cancer outcomes is a critical healthcare issue. Data from national databases clearly show that non-Hispanic whites (NHW) have a higher population-based incidence and mortality from breast cancer than Hispanic women (incidence NHW 128.1/100,000 vs. 91.9 in Hispanics; mortality NHW 21.9 vs. 14.5 in Hispanics, ACS 2015 data) [1]. On the other hand, Hispanic women with breast cancer are more likely to be younger, to present with advanced stage disease, and are more likely to have aggressive subtypes (triple negative and HER2 positive) than NHW. As a result of this, Hispanic women with breast cancer have higher mortality rates than NHW [2].

\section{Factors Contributing to Higher Breast Cancer Mortality Among Hispanics}

It has been shown that socioeconomic factors including low socioeconomic status (SES) and lack of insurance or under-insurance are the main contributors to the observed outcome disparities among Hispanic women with breast cancer [2]. A higher prevalence of obesity among Hispanics is another factor contributing to these

\footnotetext{
G. Colon-Otero $(\bowtie)$

Mayo Clinic School of Medicine Florida Campus, Jacksonville, FL, USA

e-mail: gcolonotero@mayo.edu
} 
disparities [3, 4]. Also, the prevalence of inherited BRCA mutations among different subsets of Hispanic women has been correlated with the presence of European ancestry [5]. These data suggest that the higher prevalence of aggressive subtypes of breast cancers among Hispanic women may result from interactions between environmental and genetic factors.

\section{Recent Data Elucidating the Causes of Breast Cancer Disparities Among Hispanics}

Martinez and colleagues evaluated California Cancer Registry data that consists of 29,626 Hispanics and 99,862 NHW women with invasive breast cancer who were diagnosed between 2004 and 2014 [2]. They analyzed the prevalence of different breast cancer subtypes and found that triple-negative breast cancer and HER2-positive breast cancers were more common in Hispanics (HR 1.29 and 1.19 , respectively). Hispanic women also had a higher mortality rate ratio (MMR) of 1.24. Multivariable analysis showed that neighborhood SES and health insurance status accounted for most of the differences in mortality. The percentage of Hispanic breast cancer women aged less than 50 years was twice that of NHW. Hispanics had a higher percentage of patients living in low SES neighborhoods (52.7 vs. $21.1 \%$ ) and a higher percentage of patients with Medicaid insurance (30.8 vs. 17\%). Hispanics also had more advanced (stages 3-4) disease and less stage 1 disease (39.2 vs. $28.4 \%$ and $39.8 \%$ vs. $51.1 \%$ ) and were more likely to present with positive nodes $(41.1 \%$ vs. $31.5 \%)$. Hispanics had higher TN and HER2-positive subtypes (TN: OR 1.29; ER neg HER2 pos: OR 1.31; ER pos HER2 pos: OR 1.19). In a model that included all variables, mortality among Hispanics was significantly lower than among NHW (MRR 0.90, CI 0.87-0.94). Younger women had a higher risk of dying than older women $(p<0.001)$, and mortality differences between Hispanics and NHW were greatest in the younger group (MRR 1.42 vs. 1.13).

Fejerman et al. showed that European ancestry is associated with increased risk of breast cancer among Hispanics in the San Francisco area (OR 1.79, CI 1.28-2.79) [5]. Among Mexican women, the risk of breast cancer increases with every 25\% increase in European ancestry (OR 1.20, CI 1.03-1.41) [6]. They also showed that $50 \%$ or more Native American ancestry in Hispanic women with breast cancer is associated with a doubling of mortality [7]. Engmann and colleagues in Fejerman's group recently analyzed data from the Pathways Study, a prospective study of 506 Latina women with breast cancer cared for in the Kaiser Permanente Health System. They found that equal access to care as part of the Kaiser System eliminates the association between Indigenous American ancestry and breast cancer recurrence and mortality [8]. 


\section{Breast Cancer in Young Hispanic Women: The Subset with the Greatest Disparities in Outcomes}

Even though breast cancers are most common in women in their fifth and sixth decades of life, breast cancer in women younger than 50 years is a significant health issue. For example, among US women aged 35-40 years, breast cancer is the number one cause of death and the number one cancer. Among women aged 25-34 years, breast cancer is the number two cause of death behind accidents. If we consider women aged 25-40 years, breast cancer accounts for $23 \%$ of all cancer deaths. Keegan et al. reviewed data from 5605 women aged 15-39 years that were diagnosed with breast cancer in the United States between 2005 and 2009. They found that Hispanic women had a 3.25 RR and African American (AA) women had a 1.65 RR relative to NHW women [9]. It was also found that in this age group, women with breast cancer are more likely to have triple-negative and HER2-positive disease as well as more likely to present with stages 3-4 and be Hispanic, AA, or Native American (NA) [9].

\section{Conclusions from Recent Data}

We can conclude from the recent data that breast cancer outcome disparities are greatest in the young age group (age less than 40 years) and that there is a need for more studies on the interplay between genetic and environmental factors. There is also a need for breast cancer preventive interventions in the young age group (age less than 40 years). Since low SES neighborhoods and uninsured/under-insured patients account for most of the disparities, efforts need to emphasize interventions in these populations.

\section{Hypothesis for the Causes of Early Onset Breast Cancer Disparities and the Potential Role of Childhood Obesity}

Figure 9.1 summarizes a working hypothesis for possible factors contributing to early onset breast cancer disparities. We hypothesize that low SES factors lead to increased stress and poor dietary habits, which in turn leads to childhood obesity. Childhood obesity leads to increased production of adipokines and IL-6 with increased transcription of aromatase in the adipose tissue leading to increased estrogen and increased genotoxic estrogen metabolites. This results in increased DNA damage, particularly in patients with DNA repair defects, leading to early onset breast cancer with its worse prognosis. 


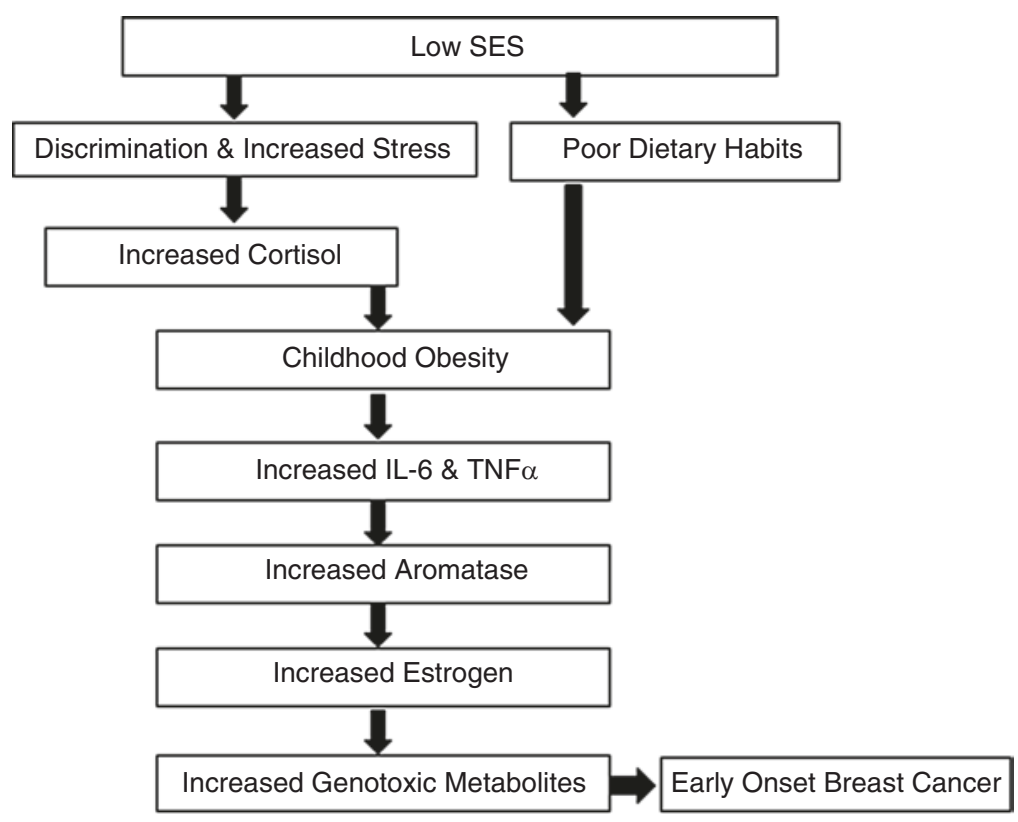

Fig. 9.1 Hispanic breast cancer outcome disparities are greatest in the early onset breast cancer subset. We speculate that this is likely a result of genetic factors and disparities in the prevalence of obesity among low SES Hispanics with its effects on estrogen metabolism

\section{Childhood Obesity: A Likely Contributor to Breast Cancer Disparities}

The prevalence of obesity in US children and adolescents has tripled over the last 30 years $[4,10]$. The prevalence of childhood obesity is highest in non-White children with 30\% AA children and 20\% Mexican American children being obese as compared to $11 \%$ NHW [4]. Recent data also shows that obesity in childhood predicts for adult obesity. Among 2400 obese 5-14 year olds followed for 17 years, $83 \%$ AA vs. $68 \%$ NHW were obese as adults [11]. Finally, low income is associated with higher obesity and with a higher consumption of fast foods, snacks, and soft drinks [4].

\section{Possible Linkage of Childhood Obesity with Breast Cancer: The Third Harvard Growth Study}

The Third Harvard Growth Study was a longitudinal study of more than 3000 school children performed by the Harvard School of Education from 1922 to 1935 [12]. The subjects were first- and second-grade public school students enrolled in 19221923 from three middle-class cities in the north of Boston. Subjects were measured annually through high school, and those with at least eight height and weight 
measurements were included in this study. Subjects were contacted in 1968 and 1988. A total of 1877 subjects were included in the analysis, including 858 women. Ever been overweight before puberty was associated with a 2.7 times risk of breast cancer mortality in females. Previous studies had yielded conflicting results regarding the risk of breast cancer associated with childhood obesity, but these studies were dependent on patients' recollection of their childhood weights [12].

\section{Childhood Obesity: A Proinflammatory State with High Estrogen and Genotoxic Estrogen Levels}

Mauras et al. evaluated a cohort of obese and lean pre-pubertal girls. In the obese girls, they found a significant increase in levels of proinflammatory cytokines, including IL-6 as well as evidence of elevated prothrombotic proteins (PAI-1 and fibrinogen) [13]. These findings show that obesity in childhood, prior to the onset of clinically evident metabolic syndrome, is associated with a significant proinflammatory and prothrombotic state. In addition, using the same cohort of patients, they showed that childhood obesity is associated with significantly higher levels of estradiol and its genotoxic metabolites (16-OH-E1, 2-OH-E1, 2-OH-E2, 4-OH-E1 and 4-OH-E2) with greatest elevations in the 16-OH-E1 levels and a significantly lower ratio of 2-OH-E1/16-OH-E1, values that had been directly and reciprocally related to the subsequent risk of breast cancer in postmenopausal women by Fuhrman et al. [14]. The elevations of estradiol and its metabolites in the obese group correlated with the elevation of IL-6.

\section{Contemporary Hormonal Contraception Is Associated with a Higher Risk of Breast Cancer}

Morch et al. recently reported a detailed epidemiologic analysis of the effects of hormonal contraceptive use on the prevalence of breast cancer. This was a prospective study of 1.8 million Danish women aged 15-49 years followed for an average of 10.9 years [15]. More than 10 years of hormonal contraceptive use was associated with a $1.38 \mathrm{RR}$ for breast cancer in this population.

\section{Oral Versus Transdermal Estrogen Replacement and Its Effects on Estrogen Metabolism}

Estrogen is converted into its oxidative metabolites in the liver, and it is conceivable that oral estrogens may be associated with a greater generation of these metabolites than the transdermal estrogens. In a prospective randomized study of hypogonadal 
girls with Turner syndrome, Dr. Mauras' team administered estradiol orally versus transdermally at doses that resulted in similar serum-free estradiol levels [12]. Measurements of total estradiol and its metabolites after 12 months of therapy were obtained. The oral estradiol group was associated with higher generation of genotoxic estrogen metabolites than normal post-pubertal girls and the transdermal estradiol group [16]. This may explain the higher risks of breast cancer associated with oral hormonal contraception reported by Morch et al. [15] and suggests that a switch from oral estrogen to transdermal estrogen contraception could potentially decrease breast cancer and ameliorate breast cancer disparities.

\section{Future Research on Genotoxic Estrogen Metabolites and Breast Cancer Disparities}

Additional studies aimed at determining if obesity in Hispanic young women is associated with higher levels of genotoxic estrogen metabolites will be of interest. Evaluating possible interactions between BRCA mutations (or other pathogenic DNA repair mutations) and genotoxic estrogen metabolism in Hispanics may identify mechanisms involved in early onset breast cancer. Short-term diet and exercise interventions may alter genotoxic estrogen metabolite generation in pre-pubertal obese girls, and measuring the effects of dietary manipulations on these metabolites may be of clinical value.

\section{Conclusions}

In summary, Hispanic women overall have lower prevalence and mortality from breast cancer than NHW. On the other hand, breast cancer in Hispanics develops at a younger age, is more likely triple-negative or HER2-positive, presents in advanced stage and has a worse prognosis. Data is emerging on the potential roles of stress from low SES status, childhood obesity, genotoxic estrogen metabolites, exogenous oral estrogens, and genetics on breast cancer, particularly early onset breast cancer and its associated disparities in Latinas. Further studies are needed to elucidate the biological factors accounting for disparities in outcome among Latinas with breast cancer.

\section{References}

1. American Cancer Society. Cancer facts \& figures for Hispanics/Latinos 2015-2017. Atlanta: American Cancer Society; 2015.

2. Martínez ME, Gomez SL, Tao L, Cress R, Rodriguez D, Unkart J, et al. Contribution of clinical and socioeconomic factors to differences in breast cancer subtype and mortality between 
Hispanic and non-Hispanic White women. Breast Cancer Res Treat. 2017;166(1):185-93. https://doi.org/10.1007/s10549-017-4389-z.

3. Patel TA, Colon-Otero G, Bueno HC, Copland JA, Perez EA. Breast cancer in Latinas: gene expression, differential response to treatments, and differential toxicities in Latinas compared with other population groups. Oncologist. 2010;15(5):466-75. https://doi.org/10.1634/ theoncologist.2010-0004.

4. Caprio S, Daniels SR, Drewnowski A, Kaufman FR, Palinkas LA, Rosenbloom AL, et al. Influence of race, ethnicity, and culture on childhood obesity: implications for prevention and treatment: a consensus statement of Shaping America's Health and the Obesity Society. Diabetes Care. 2008;31(11):2211-21. https://doi.org/10.2337/dc08-9024.

5. Fejerman L, John EM, Huntsman S, Beckman K, Choudhry S, Perez-Stable E, et al. Genetic ancestry and risk of breast cancer among U.S. Latinas. Cancer Res. 2008;68(23):9723-8. https://doi.org/10.1158/0008-5472.CAN-08-2039.

6. Fejerman L, Romieu I, John EM, Lazcano-Ponce E, Huntsman S, Beckman KB, et al. European ancestry is positively associated with breast cancer risk in Mexican women. Cancer Epidemiol Biomark Prev. 2010;19(4):1074-82. https://doi.org/10.1158/1055-9965.EPI-09-1193.

7. Fejerman L, Hu D, Huntsman S, John EM, Stern MC, Haiman CA, et al. Genetic ancestry and risk of mortality among U.S. Latinas with breast cancer. Cancer Res. 2013;73(24):7243-53. https://doi.org/10.1158/0008-5472.CAN-13-2014.

8. Engmann NJ, Ergas IJ, Yao S, Kwan ML, Roh JM, Ambrosone CB, et al. Genetic ancestry is not associated with breast cancer recurrence or survival in U.S. Latina women enrolled in the Kaiser Permanente Pathways study. Cancer Epidemiol Biomark Prev. 2017;26(9):1466-9. https://doi.org/10.1158/1055-9965.EPI-17-0148.

9. Keegan TH, DeRouen MC, Press DJ, Kurian AW, Clarke CA. Occurrence of breast cancer subtypes in adolescent and young adult women. Breast Cancer Res. 2012;14(2):R55.

10. Ogden CL, Flegal KM, Carroll MD, Johnson CL. Prevalence and trends in overweight among US children and adolescents, 1999-2000. JAMA. 2002;288(14):1728-32.

11. Freedman DS, Khan LK, Serdula MK, Dietz WH, Srinivasan SR, Berenson GS. Racial differences in the tracking of childhood BMI to adulthood. Obes Res. 2005;13(5):928-35.

12. Mauras N, DelGiorno C, Kollman C, Bird K, Morgan M, Sweeten S, et al. Obesity without established comorbidities of the metabolic syndrome is associated with a proinflammatory and prothrombotic state, even before the onset of puberty in children. J Clin Endocrinol Metab. 2010;95(3):1060-8.

13. Mauras N, Santen RJ, Colón-Otero G, Hossain J, Wang Q, Mesaros C, et al. Estrogens and their genotoxic metabolites are increased in obese pre-pubertal girls. J Clin Endocrinol Metab. 2015;100(6):2322-8.

14. Fuhrman BJ, Schairer C, Gail MH, Boyd-Morin J, Xu X, Sue LY, et al. Estrogen metabolism and risk of breast cancer in postmenopausal women. J Natl Cancer Inst. 2012;104(4):326-39. https://doi.org/10.1093/jnci/djr531.

15. Mørch LS, Skovlund CW, Hannaford PC, Iversen L, Fielding S, Lidegaard Ø. Contemporary hormonal contraception and the risk of breast cancer. N Engl J Med. 2017;377(23):2228-39. https://doi.org/10.1056/NEJMoa1700732.

16. Mauras N, Torres-Santiago L, Santen R, Mericq V, Ross J, Colon-Otero G, et al. Impact of route of administration on genotoxic oestrogens concentrations using oral vs transdermal oestradiol in girls with Turner syndrome. Clin Endocrinol. 2019;90(1):155-61. https://doi. org/10.1111/cen.13869. 
Open Access This chapter is licensed under the terms of the Creative Commons AttributionNonCommercial 4.0 International License (http://creativecommons.org/licenses/by-nc/4.0/), which permits any noncommercial use, sharing, adaptation, distribution and reproduction in any medium or format, as long as you give appropriate credit to the original author(s) and the source, provide a link to the Creative Commons license and indicate if changes were made.

The images or other third party material in this chapter are included in the chapter's Creative Commons license, unless indicated otherwise in a credit line to the material. If material is not included in the chapter's Creative Commons license and your intended use is not permitted by statutory regulation or exceeds the permitted use, you will need to obtain permission directly from the copyright holder. 